\title{
Féeries
}

Études sur le conte merveilleux, XVII $-\mathrm{XIX}{ }^{\mathrm{e}}$ siècle

\section{Robes classiques et robes symbolistes, de «Peau d'Âne » aux avatars de « Barbe bleue »}

Classical and Symbolist Dresses, from "Donkey Skin" to "Blue Beard's" Avatars

Jean-Paul Sermain

\section{(2) OpenEdition}

1 Journals

Édition électronique

URL : http://journals.openedition.org/feeries/1285

DOI : $10.4000 /$ feeries. 1285

ISSN : 1957-7753

Éditeur

UGA Éditions/Université Grenoble Alpes

Édition imprimée

ISBN : 978-2-37747-075-4

ISSN : $1766-2842$

Référence électronique

Jean-Paul Sermain, «Robes classiques et robes symbolistes, de «Peau d'Âne » aux avatars de "Barbe bleue » », Féeries [En ligne], 15 | 2018, mis en ligne le 14 février 2019, consulté le 10 décembre 2020. URL : http://journals.openedition.org/feeries/1285; DOI : https://doi.org/10.4000/feeries.1285

Ce document a été généré automatiquement le 10 décembre 2020.

(c) Féeries 


\section{Robes classiques et robes symbolistes, de « Peau d'Âne " aux avatars de «Barbe bleue »}

Classical and Symbolist Dresses, from "Donkey Skin" to "Blue Beard's" Avatars

Jean-Paul Sermain

Le conte de fées, ce genre de récit court teinté de merveilleux né à la fin du XVII siècle en France et se développant sur sa lancée au long du XviII ${ }^{e}$ siècle, avant les transformations majeures subies au XIX ${ }^{\mathrm{e}}$ siècle à partir de l'Allemagne romantique, estil à même d'exprimer et d'utiliser "l'univers sensible" différemment ou plus largement que les autres types de récits fictionnels de la même époque? Sur cette question poétique, Christelle Bahier-Porte et Emmanuelle Sempère en greffent une autre plus historique, en invitant à mettre en rapport une présence sensible dans le conte avec le nouveau statut de l'expérience des sens dans la philosophie instaurée par John Locke, et dans le tournant scientifique fondé sur l'observation des faits (mais il faudrait ajouter aussi sur les hypothèses et les calculs selon les principes qui restent fondamentaux du cartésianisme, voir les Pensées sur l'interprétation de la nature de Diderot en 1753). Il est difficile d'établir des liens entre un ensemble de textes écrits entre 1690 et 1700 - et qu'on peut qualifier de «classique » en nous conformant à l'usage critique d'aujourd'hui et au rôle que jouent dans sa formation les conceptions de la langue et de l'œuvre littéraire propres à la génération antérieure -, et une théorie de la connaissance et de l'homme qui ne s'impose en France qu'à partir des années 1740 , d'autant plus que le genre du conte de fées, dans cette décennie fondatrice de la fin du XVII ${ }^{\mathrm{e}}$ siècle, obéit à des intentions spécifiques et se situe dans des traditions propres aux différents domaines de la fiction et de la fable ${ }^{1}$. L'attention au sensible du premier conte de fées français mérite sans doute d'être rapprochée aussi du style des représentations picturales et plastiques contemporaines depuis lors reconnu et compris avec la notion de rococo : le monde s'y offre dans ses couleurs, sa sensualité, ses plaisirs en utilisant de façon assez désinvolte les situations qui les amènent. Une telle économie n'est-elle pas aussi propre à ce petit genre de récit? Toutefois, les 
rapports entre des choix esthétiques assez évidents à Paris ou à Venise puis à Londres et en Bavière, et la littérature française sont eux-mêmes difficiles à établir et finalement assez réduits, dans la mesure où ce qui définit un style pictural ne saurait se retrouver dans un style de récit, à moins de se contenter d'analogies entre des modes spécifiques de la représentation. Tout en gardant à l'esprit ces différentes approches du phénomène, on peut ainsi essayer de répondre à la question de l'articulation entre le genre du conte et un mouvement de pensée qui devient dominant (mais jamais exclusif), par une attitude modeste, en se plaçant en retrait, à l'intérieur du champ des fictions à caractère féerique et en considérant comment y est introduit, représenté, transmis l'univers sensible.

1 Ce qui frappe au préalable et qui peut servir comme d'arrière-fond contrastif au conte de fées, c'est combien la littérature de fiction de la fin du règne de Louis XIV jusqu'au milieu du siècle suivant (environ) a pris une orientation qui évacue ou filtre sévèrement le monde sensible, $\mathrm{du}$ moins s'intéresse principalement à sa résonance dans la conscience et plus encore dans la réflexion - parce qu'est privilégié le compte rendu écrit à distance par le personnage de ce qu'il a vécu (dans les deux formes empruntées des mémoires et du recueil épistolaire), et que l'intérêt se focalise sur la vie intérieure et les discussions des personnages, selon la voie ouverte par $\mathrm{M}^{\text {lle }}$ de Scudéry, ce qu'elle complétait néanmoins par des combats et par des descriptions, les uns et les autres ensuite écartés, comme l'avait demandé en ricanant Boileau. Ce qui intéresse les romanciers comme Marivaux, Crébillon et Prévost, c'est la transformation de l'expérience troublée en un discours qui la clarifie, la pense, la communique: la représentation romanesque est nourrie des représentations que les personnages élaborent d'eux-mêmes et de leurs partenaires (l'œuvre apparaissant alors comme une représentation oblique de représentations). Réverbérations lointaines que complètent à peine, tant sont-elles elles-mêmes assagies et mises à distance, les notations comiques où le monde sensible est retenu par ce qu'il a de blessant (pour le corps abîmé et le corps humilié). Dans la seconde moitié du xvIII ${ }^{e}$ siècle, Diderot et Rousseau, qui se consacrent à des expériences sensibles inédites (paysage, promenade, jardin, tableau, amour, musique etc.), tendent à les justifier par des réflexions et des commentaires. La lettre sur le Valais dans la Nouvelle Héloïse (I, 23) décrit moins qu'elle n'explique en quoi consiste le paysage de montagne par exemple, ses composantes, ses bienfaits, ses caractéristiques : elle enseigne!

2 L'évolution n'est certainement pas linéaire qui conduit à la rupture saisissante des poèmes de Wordsworth écrits dans la dernière décennie $d u x{ }^{e}{ }^{e}$ siècle, ou ceux de Keats et de Shelley une vingtaine d'années plus tard conjointement aux romans de Scott. Cela vaut également en sens inverse, dans ce qui conduit au conte de fées. Arrêtons-nous soixante-dix ans avant l'émergence des contes classiques français et restons un moment avec un des critiques (et traducteurs) les plus remarquables (et en même temps négligé) dans l'appréhension des genres fictionnels au XVII siècle, Jean Chapelain (1595-1674). En 1619 et 1620, il place une "Épître dédicatoire et avertissement au lecteur " en tête de sa traduction de la première puis de la seconde partie du roman de Mateo Alemán, Le Gueux ou la vie de Guzman d'Alfarache, image de la vie humaine. En 1623, il publie la Préface de l'Adone de Marin, en tête de la première édition de cette œuvre, parue à Paris en italien ; vingt ans plus tard, en 1646, il aura rédigé « De la lecture des vieux romans, dialogue »(publié seulement en 1728) ${ }^{2}$. Les trois premiers textes presque contemporains (augmentés des deux volumes de la traduction 
d'Alemán) témoignent d'une extraordinaire attention à ce que la fiction propose de son temps de plus neuf et de plus original. Il présente l'œuvre d'Alemán comme une "satire» et renvoie à son "prototype " Lazarille de Tormès. Le roman picaresque espagnol ouvrait une voie inédite, d'une part en découvrant dans la réalité contemporaine vue dans toute son ampleur (c'est-à-dire jusque dans sa matérialité la plus infime) une matière romanesque inédite, d'autre part en créant une continuité dans les épisodes (qui pouvaient se nourrir de la tradition des nouvelles) par l'instauration d'un point de vue qui agit à la fois comme conscience et comme écriture, l'une et l'autre dédoublées entre le temps du vécu et celui du remémoré. La préface à l' Adone de Marin qualifie cette œuvre par la nouveauté de son invention, il s'agit d'une "épopée de paix» qui se caractérise par un registre moyen capable d'associer le comique, le tragique et l'héroïque, donc un mélange des registres; Chapelain la voit s'épanouir surtout dans les descriptions qui donnent libre champ à un «style " remarquable (il définit aussi de façon très intéressante le style du livre espagnol que le traducteur doit garder : « Cet esprit de vie qui en entretient et anime les membres ; car le style est l'âme du discours et ce qui l'informe ${ }^{3}$.») Envisageant différents types d'invention, il oppose les inventions contre nature aux naturelles. Les premières se produisent "lors qu'à un corps d'une nature, un autre corps d'une autre nature est conjoint, comme on a vu des satyres "; et Chapelain donne pour exemple « les rêveries et contes de nourrices à leurs enfançons, ou si vous voulez une partie des nouvelles de Straparole, auteur italien, dans lesquelles sans nécessité d'allégorie, il fait parler et agir les animaux irraisonnables comme parlent et agissent les hommes » (Opuscules critiques, p. 102). Les autres inventions greffent sur un corps d'une nature une partie d'un autre corps de même nature (ainsi d'un homme à deux têtes, ou les hermaphrodites, ou les " enfants attachés par le front»). De cette sorte sont «les romans en général de toute espèce, qui n'ont point ou unité d'action ou unité de personnes agissantes " (leur dispersion fatigue: il suffit d'en ouvrir un de cette époque pour s'en convaincre). L'invention naturelle est celle des arts et des sciences ou, au second niveau, "l'invention des espèces » comme l'héroïque, le lyrique. Se situe à ce second niveau, inférieur, l'épique de paix cultivé par Marin. Chapelain saisit donc les faiblesses des romans de son temps (peut-être ne met-il pas dans cette catégorie l'Astrée, lue comme une pastorale ?), mais aussi ce qu'a d'original le recueil de Straparole : il dessine déjà le champ du conte de fées à venir. Dans le dialogue plus tardif « De la lecture des vieux romans ", il analyse la configuration historique permettant de penser ce futur genre et plus particulièrement sa poétique. Il conçoit les modalités d'une lecture postcritique des romans de chevalerie décriés: ils ont une valeur de témoignage culturel et manifestent une certaine disposition de l'esprit dans l'attachement à des valeurs morales et sociales. Le merveilleux prend un sens littéraire et anthropologique au second degré (ce que retiendra aussi la critique anglaise réhabilitant les romans de chevalerie dans la seconde moitié du XVIII ${ }^{\mathrm{e}}$ siècle) : le genre du conte de fées naîtra de l'articulation de ce principe poétique et moral trouvé dans les romans de chevalerie sur le type de récits cultivé par Straparole. Chapelain fournit les deux clefs dont s'emparera Perrault ensuite 4 .

3 Ajoutons à cela que les deux textes étrangers que Chapelain introduit (et pour l'un traduit), l'épopée de paix de Marin et la satire picaresque d'Alemán offrent deux modes de traitement stylistique du monde réel qu'ils développent dans toute sa diversité et avec toutes les intensités de la sensibilité. Ils sont évidemment aux antipodes l'un de l'autre: l'un enchantant le réel dans le déploiement poétique des descriptions et des 
évocations sensuelles prises dans la surenchère luxueuse que permet la mythologie, l'autre faisant de la rencontre du réel comme un choc toujours douloureux et scandaleux, conformément au projet d'une critique véhémente déployée dans une écriture savante. Marino comme Alemán tendent en effet à saisir la relation au monde sensible dans les rets d'un conceptisme théorisé à l'époque ${ }^{5}$, dont le second XVII siècle se détachera avec véhémence et orgueil, et singulièrement le conte de fées porté par une sorte d'épiphanie de la simplicité populaire. L'une des contributions les plus intéressantes pour notre propos de la préface de l'Adone est la place fondamentale accordée à l'émotion dans deux développements que Chapelain n'a pas cherché à concilier - en partie pour ne pas exaspérer les lecteurs religieux dont il a vu la hargne s'exercer sur Galilée -, et c'est à eux qu'il pense en mettant l'accent sur le bénéfice de "purgation » de cette production d'émotion. La "créance " que l'on peut donner à l'histoire est essentielle, «où la créance manque, l'attention ou l'affection manque aussi, mais où l'affection manque il n'y a point d'émotion » (p. 111). Chapelain dénonce les romans qui suscitent «l'amusement des idiots et l'horreur des habiles », pour ne pas être capables d'émouvoir : "pour émouvoir il faut faire impression ", il précise ensuite que la "purgation" «ne s'obtient que par le seul plaisir", "de telle sorte que sans plaisir il n'y a point de poésie ». Le comble du plaisir naîtra de «l'assemblement judicieux » des choses et des descriptions : la présence du monde sensible dans son articulation à la matière narrative est donc source d'émotions et de plaisirs, Chapelain n'ose pas dire du plaisir de l'émotion (mais laisse le lecteur y venir). Un vers de l'Adone $(\mathrm{V}, 136)$ nous en donne une idée, il concerne un "intermède " placé dans la représentation théâtrale du malheur d'Actéon offerte à Adonis. C'est le spectacle d'une bataille : « Armonica e per arte è la battaglia » ("Artificielle et rythmée est la bataille »), et le choc des armes et des hommes «fan di mezo a l'orror nascer diletto " ( font au milieu de l'horreur naître le plaisir »), écho de deux vers du Tasse, Gerusalemme liberata (XX, 30) : «Bello in si bella vista anco è l'orrore,/E di mezo la tema esce il diletto » (" Devient belle dans une image si belle même l'horreur de la guerre, et au sein de la crainte naît le plaisir »). Le conte de Perrault l'emporte par la «créance » qu'il garantit en faisant naître lui aussi, de l'horreur et de la crainte, le plaisir : pensons au Petit Poucet et à ses frères dans la forêt et dans la maison de l'ogre, à Riquet, au fils du meunier, à Cendrillon ${ }^{6}$.

4 Marino s'efface pourtant à la fin du $\mathrm{XVII}^{\mathrm{e}}$ siècle, comme Lesage doit traduire le picaresque pour le mettre au goût du jour. Ce retour en arrière laisse entrevoir ce dont la fiction classique se sépare pour de nouveaux terrains, mais aussi ce dont le conte de fées entend faire résonner le souvenir, puisque les fictions anciennes, « vieux romans ", "contes de nourrices", "épopée de paix», "satire», viennent heureusement alimenter ce qui doit tenir lieu de culture archaïque et populaire, en donner comme le truchement pour un public cultivé (plus que lettré), et ainsi créer l'impression d'une origine lointaine et d'une conformation du merveilleux à une disposition anthropologique ancienne et finalement elle-même merveilleuse dans sa naïveté, mais irrémédiablement le propre de l'homme. Le conte de fées classique, à son meilleur, est donc capable de reprendre des traits poétiques antérieurs, de jouer de leur distance, tout en les soumettant à la logique du récit bref et entièrement porté par la dynamique de l'action, qui est sa façon d'intégrer les nouveaux idéaux d'expression et de formation littéraire du second XVII siècle (la catégorie de "classico baroque " défendue par Hélène Merlin-Kajman ${ }^{7}$ pourrait là trouver une application, à moins qu'on ne préfère identifier ce qu'elle repère au rococo). Ce croisement peut être apprécié justement dans 
ce qui était un des aspects majeurs des deux grands textes introduits en France par Chapelain comme de ceux qu'il a évoqués (pour désavouer les premiers et racheter les seconds, contes de nourrices, vieux romans), c'est-à-dire dans la figuration du monde sensible, dont l'intensité tenait à l'expression passionnée d'un rapport personnel fasciné, tout de séduction et d'érotisme chez Marin, de cruauté et de répulsion chez Alemán. Le style désormais, avec Perrault et ses amies conteuses, ne passera plus par un travail ostentatoire sur la langue ni par la véhémence, mais par une forme de récit et par des effets de connotation familière obtenus principalement par la désuétude des termes et des situations narratives.

5 Chez Perrault, le monde sensible s'offre dans une extension remarquable pour un recueil si bref. Il obéit à la logique "bizarre et frivole ${ }^{8}$ " du conte, ce que le mot de burlesque dans son acception moderne retrouve assez bien, et en même temps il s'intègre à l'expérience pathétique des personnages, à leurs émotions dont PierreEmmanuel Moog étudie ici les médiations. Tout élément sensible intervient en effet sur deux registres du récit: il est un élément comme extérieur offert à l'attention du lecteur et auquel il réagit directement (ainsi des descriptions panoramiques de Balzac ou Stendhal), mais il est aussi présent à travers l'implication du personnage, et le lecteur se laissera alors toucher selon qu'il se sent proche ou non de lui (d'où l'importance cruciale de la « créance » selon Chapelain, et la supériorité de Perrault sur ses amies conteuses). Notons que la robe et le coffre de Marianne dans le roman de Marivaux ne sont pas présentés physiquement au lecteur (il ne peut s'en faire aucune idée) et valent par les réactions de l'héroïne, le sens qu'elle leur donne, ses propres émotions, assez intenses, ou celles qu'elles suscitent (l'émoi érotique de $\mathrm{Climal}^{9}$ pour la robe, le sentiment d'une communauté pour $\mathrm{M}^{\mathrm{me}}$ de Miran).

6 La frivolité se situe aux deux bouts du plus prosaïque et du merveilleux. Ainsi dans «Peau d'Âne ${ }^{10}$ ", l'héroïne glisse comme signal dans le gâteau fait pour le prince un anneau qu'il a failli avaler, fatale erreur. On peut penser aux petits cailloux ou aux miettes de pain du Petit Poucet $^{11}$. Mais la pantoufle de verre transforme merveilleusement la réalité triviale, comme les bottes chaussées par le chat, les bottes de sept lieues, la clef qui devient signe naturel (par la tâche de sang indélébile), et singulièrement les quatre robes de Peau d'Âne qui vont nous occuper ici. Obtenues par des moyens merveilleux mais sales (l'excrément de l'âne), les trois premières sont sollicitées pour éviter l'inceste et données pour y parvenir : elles sont prises dans une lutte de type tragique. Leur caractère unique et fabuleux tient tout entier dans la formule qui les nomme (et que le cinéma de Jacques Demy exploitera, tout comme Diderot imagine le ciel directement reproduit sur la toile du peintre Vernet, autre magie). À l'instigation d'une fée marraine, l'héroïne veut d'abord une robe « couleur du temps ", ce qui est bien mystérieux, la robe y répond un peu avec son «bleu de l'Empyrée [...] ceint de gros nuages d'or ». Elle demande ensuite une robe "couleur de la lune », et la lune se fait robe: "Dans les Cieux où la Nuit a déployé ses voiles, / La lune est moins pompeuse en sa robe d'argent / Lors même qu'au milieu de son cours diligent / Sa plus vive clarté fait pâlir les étoiles. » Elle demande en troisième lieu une robe «couleur du soleil » et Perrault décrit la dernière prouesse: «[...] l'ouvrage précieux, / Si beau, si vif, si radieux, / Que le blond amant de Clymène, / Lorsque sur la voute des Cieux / Dans son char d'or il se promène, / D'un plus brillant éclat n'éblouit pas les yeux. » Perrault applique à la robe le motif traditionnel de l'éloge féminin : c'est que la robe, pour le père, livrera la femme. Ces sommets de la merveille paralysent l'hérö̈ne et ne l'enchantent pas. Le texte joue donc d'une contradiction entre l'éclat 
produit (qu'on pourrait relier à Marino) et sa valeur pour le personnage (plus proche de l'univers d'Alemán). On se rapproche encore plus d'Alemán avec le quatrième vêtement, la peau d'âne. Elle "épouvante » l'héroïne qui en tire son nom, la fée y voit «un masque admirable»: "On ne croira jamais, tant elle est effroyable, / Qu'elle renferme rien de beau.» Peau d'Âne se présente désormais couverte "d'une vilaine crasse ", "maussade et pleine d'ordure ", c'est une "sale créature». Le lecteur est invité ici à partager l'horreur ressentie au contact physique de ce vêtement abominable et face aux violences qu'il suscite - l'héroïne est "harcelée » de "quolibets » et de «bons mots ", elle est " tiraillée » et " raillée ». Le comportement méchant (propre au picaresque) se greffe sur une situation et un habit extraordinaires qui offrent une image sensible d'une chute aux limites de l'humanité, ce que dit dans d'autres contes le terme de « monstre ", appliqué aux héros du « Riquet » et à de nombreux personnages des contes féminins, ou encore la situation de Cendrillon ${ }^{12}$.

7 Le dimanche, Peau d'Âne s'enferme dans sa chambre (tentant ainsi de s'approprier sa relégation), et, s'étant décrassée, met tour à tour chacune des trois robes et se contemple dans un grand miroir (peu vraisemblable ici) : elle finit par se laisser toucher par ce qui avait peut-être déjà séduit le lecteur, qui peut désormais légitimement partager «l'impression » des robes et en être lui aussi «ému » (pour reprendre les termes de Chapelain). La jeune fille remarque le fils du roi de passage à la ferme, lui aussi lié au soleil par la comparaison à Céphale. Le vers «Elle gardait encore le cœur d'une Princesse » peut faire penser à la situation de Théodore ${ }^{13}$ de Corneille, qui résume sa situation ainsi : «Dans mon sort ravalé, je sais vivre en princesse [...]. » Un dimanche, au moment où la jeune fille met la robe couleur du soleil ${ }^{14}$, le prince, allant au fond des basses-cours, la surprend en se penchant « au trou de la serrure ». Un nouveau regard vient saisir la robe, c'est un regard de « désir » érotique, fixé sur la personne qui la revêt. Le vêtement, d'abord ramené à sa fonction corruptrice, devenu un moyen de se voir et de restaurer la confiance en soi (l'évocation sensible rend alors compte de cette nouvelle situation), prend enfin sa pleine valeur sur le corps de la jeune fille et sa description est intégrée à une action, exactement un mouvement amoureux. Ce rapport au corps prend même la forme d'un très bref drame. Le prince en tire un plaisir paroxystique (il « ne peut qu'à peine, / En la voyant reprendre haleine, / Tant il est comblé de plaisir »-celui-ci s'apparente donc à l'émotion sexuelle la plus intense); il est tenté de forcer la porte ( Trois fois dans la chaleur du feu qui le transporte, / Il voulut enfoncer la porte »), et le contexte culturel invite à penser qu'il comptait ainsi forcer une jeune fille isolée, qui fait la faute de porter la robe, un habit de séduction publique, dans un lieu où elle est sans défense; elle séduit là où elle ne le devrait pas, comme le petit chaperon rouge semblable à l'agneau de La Fontaine ayant quitté le troupeau et la protection des chiens. Mais le drame amorcé connaît une bonne fin : les charmes de la jeune fille qui asservissent le cœur du héros deviennent « Des beautés de son âme, assuré témoignage ». Ce qui réoriente le mouvement libidinal du prince est qu'il découvre ce qu'il y a de « divin » dans la jeune femme, c'est-à-dire une âme dont la beauté tient à son origine surnaturelle. La femme, un moment reléguée aux limites de l'humanité, est pleinement reconnue sur deux registres bien distincts. D'un côté, cette robe de princesse montre une princesse, elle confirme une identité sociale, le conte imaginant un monde où la robe royale se distinguerait par son luxe inouï de toutes les autres - chacun doit porter des habits qui annoncent sa place, comme le réclament beaucoup d'utopies contemporaines. Cette robe était nécessaire pour que le fils du roi remarque la princesse et éprouve d'emblée une passion violente. Cette robe a aussi 
permis qu'elle ne soit pas traitée en bête, et cette dégradation serait venue, nous suggère le texte, d'un viol : une fille de rien dans un lieu isolé l'appelle et le justifie, surtout si elle exhibe sa beauté et s'y complait. Mais le viol ne se produit pas parce que le prince ne s'arrête pas à la robe et à la mise en valeur séduisante du corps (ce que retient Marivaux dans sa reprise de l'épisode avec sa Marianne): la robe aide à retrouver ce que la femme a de plus précieux, « les beautés de son âme ", c'est-à-dire ce qui en elle ne lui est pas propre, et pourrait-on dire pas même individuel, mais est un don de Dieu accordé à tous les hommes. Cette empreinte du Créateur peut être offusquée dans le régime de la nature déchue: le père se fondait sur une fausse ressemblance avec la mère et une beauté sidérante, pour vouloir épouser sa fille; les paysans de la ferme prolongent le trouble initial en assimilant la jeune fille à son autre robe, la peau d'âne, et en ignorant tout autant sa juste identité ; le prince découvre le corps somptueux et est prêt à s'en emparer pour mener à son sommet son plaisir - déjà presque à son comble! Après ces trois égarements affreux, la robe sert finalement à rendre visible la vraie nature de la jeune femme.

8 La robe a donc une présence sensible (il faudrait dire l'ensemble des quatre robes), qui s'impose à la fois dans sa splendeur et dans son horreur (les deux registres de Marino, et l'un des registres picaresques), et elle suscite différents investissements des personnages et par contrecoup du lecteur, qui en déterminent les valeurs. Celles-ci sont dramatiques, c'est le plus évident, elles sont morales (on vient de le voir) et elles viennent nourrir l'herméneutique plus fine du lecteur parce qu'elles se situent, singulièrement, à la jointure mystérieuse du corps et de l'âme. Autant dire que dans sa matérialité, la robe sert de médiation à une métaphysique qui ne peut s'appréhender sans le corps, sans le signe, sans le langage sensible. L'âme est indissociable du corps, $\mathrm{du}$ vêtement qui le couvre et qui a un prix, comme de sa position et sociale et accidentelle, mais elle le transcende, elle impose le respect et une vision qui le dépasse, tandis que le corps, d'une beauté si touchante, paraît trompeusement se suffire à luimême, dans une autonomie dont l'inceste et le viol disent la vérité.

Perrault combine ce qu'il y a de plus prosaïque, avec les mouvements les plus élevés du tragique et du religieux - le lien à Corneille n'est peut-être donc pas fortuit. La princesse est aussi une jeune "souillon " qui compense sa dégradation physique et affective le dimanche par la rêverie et le travestissement; le prince agit en homme sensuel, en chasseur, et se convertit à l'amour chaste au fond des basses-cours (il est proche d'un autre prince face à la Belle au bois dormant)! Le monde sensible n'est ni célébré dans sa brillance baroque ni provocant dans son toucher picaresque, pas plus qu'il n'est source d'un savoir - selon l'épistémologie sensualiste -, mais le propre du conte de fées permet à la fois de le vivre dans sa matérialité immédiate et d'en manifester les implications subjectives, morales, sociales et même métaphysiques ${ }^{15}$.

La singularité de cette conduite du récit merveilleux pourrait être mesurée par une comparaison avec le roman de la génération suivante, je l'ai amorcée en reliant la robe de Peau d'Âne à celle que revêt Marianne dans le roman de sa vie par Marivaux, où l'âme n'est plus le miroir du Créateur mais affleure dans une relation intersubjective, donc non plus verticalement mais horizontalement - même si Marivaux insiste conjointement sur la verticalité des rapports sociaux, comme il socialise entièrement le viol ou du moins la violence propre à la prostitution. La robe corruptrice devient l'instrument d'une reconnaissance et est même indispensable, ce que le texte de Perrault laisse comprendre sans le dir ${ }^{16}$. Je me propose de compléter ce dialogue des 
textes en considérant la reprise des contes de Perrault dans un autre temps et un autre contexte, celui du symbolisme français. Il faudra pour cela faire un quart de tour, puisque «Peau d'Âne » n'inspire pas les écrivains de la dernière partie du XIX ${ }^{e}$ siècle, et considérer les avatars de "Barbe bleue ${ }^{17}$ ", beaucoup plus nombreux, et surtout qui vont en quelque sorte greffer le motif des robes venu de «Peau d'Âne » dans la relation du héros non plus avec sa seule épouse mais avec ses épouses. Nous évoquerons comment deux auteures d'aujourd'hui ont interprété et repris récemment cette bascule symbolique dans leur propre réécriture de «Barbe bleue ».

11 La «Barbe bleue » de Perrault donne aux objets luxueux de la vie moderne la première place, il les présente même comme une des motivations de l'héroïne pour accepter le mariage avec le héros inquiétant, puis de la curiosité qui la fait parcourir furieusement l'hôtel de son mari dès qu'il est parti. Mais s'ils sont nommés, ils ne sont pas le support d'une description. La chambre interdite n'en recèle aucun, contrairement à ce que la quête de l'héroïne laissait attendre, mais à leur place le cruel savoir d'une nature martyrisée, les corps sanglants des épouses précédentes (là aussi contre toute vraisemblance).

12 L'attrait des biens matériels et l'obsession de leur possession, qui sont au principe du développement industriel de l'économie au XIX ${ }^{\mathrm{e}}$ siècle, Flaubert les inscrit au cœur de Madame Bovary et de L'Éducation sentimentale; les auteurs symbolistes les privilégient à leur tour dans leurs adaptations des contes de Perrault, et singulièrement dans leur reprise de la «Barbe bleue ». France et Daudet lui donnent un tour comique. Henri de Régnier pas du tout et il s'éloigne de la simplicité des contes pour adopter le rythme de la nouvelle (ce «conte» paraît en 1892 dans la revue des Entretiens politiques et littéraires, il est repris en 1893 dans le recueil Contes de soi-même, lui-même intégré à $L a$ Canne de jaspe en $1897^{18}$ ). Le titre annonce clairement une version supplémentaire d'un conte de fées classique: "Le sixième mariage de Barbe bleue» (notons que le personnage de Perrault s'appelle «la Barbe bleue » et qu'il est mentionné seulement " plusieurs femmes », et « on ne savait ce que ces femmes étaient devenues »).

13 Les trois premiers paragraphes installent le lecteur au cœur d'une église gothique, avec une attention aux détails, aux couleurs, aux lumières, aux sons, aux parfums, totalement étrangère aux contes classiques. L'auteur du récit se manifeste ensuite dans une évocation, tout aussi riche en notations visuelles et sensorielles, de la ville, de ses habitants et de la campagne. Dans la promenade en bateau qu'il s'offre ensuite, il introduit le mouvement dans sa description. Il fait défiler les deux rives et étoffe leur représentation mobile par des métaphores (ainsi des «porches d'ombre " le long de l'eau, des "grottes ») et par des allusions culturelles (ainsi de la rivière du Styx), en mettant toujours l'accent sur l'atmosphère (ainsi est-il pris d'« angoisse » le soir venu). Le promeneur lassé du spectacle s'apprête à partir quand il découvre une petite maison et entre en dialogue avec une vieille femme qui cherche à vendre son logis proche $\mathrm{du}$ château de Barbe bleue fort couru des curieux. Par un glissement, cette femme lui apparaît comme " contemporaine du sire légendaire » et il évoque la mort des épouses. À son tour il découvre la ruine du château dont il ne reste que la tour où est montée sœur Anne. L'ultime description de la promenade évoque les «ombres" «douces, mélancoliques et nues des épouses ». Commence alors, sans autre transition, une seconde partie du texte qui installe le lecteur dans le passé et dans le château de Barbe bleue encore intact. Ce changement d'univers sensible annonce l'innovation essentielle de Régnier: les robes ont suscité à la fois la demande matrimoniale et le geste 
meurtrier. Aussi le récit recense avec force détails les cinq robes distinguées par leur couleur : les quatre premières, blanche, bleue, mauve, vert, sont associées aux éléments naturels qui permettent d'enrichir leur pouvoir de suggestion ; la cinquième évoque la robe couleur du temps de "Peau d'Âne »: «Une enfin, la cinquième, s'enveloppait d'une pièce de mousseline ample et si légère qu'en se superposant ou en se dédoublant elle paraissait selon son épaisseur ou sa transparence de la couleur de l'aube ou du crépuscule ${ }^{19}$. " Le conte nous dit alors que le seigneur les a aimées, puis introduit une nouvelle liste, celle des demeures familiales laissées par les cinq jeunes femmes, toutes évidemment très pittoresques. La clef du mystère nous est ensuite donnée : «[...] il ne les aima que pour leurs robes variées ces épouses [...], et sitôt qu'elles avaient façonné les étoffes qui les vêtaient [...], il tuait d'une main cruelle et sage les Belles inutiles. » (p. 440) Il conserve chacune des robes dans " une chambre spéciale », dont il choisit le mobilier et le parfum et qui font «transpirer» des musiques, source d'une nouvelle liste fort ample. Le conte s'éloigne alors du château et s'adresse à une bergère éloignée de tout et ignorante de ces mariages antérieurs, elle est proche, par sa position, de la Grisélidis et de la vieille femme avec son rouet dans le château familial de celle qui n'est pas encore au bois dormant. Elle porte seulement « une cape de laine » et une "coiffe de toile ». Barbe bleue veut l'épouser. Suit le récit de son mariage avec la fanfare, le cortège, le peuple amateur de "faste »; le vieux seigneur vient chercher sa belle dans la chaumière, qui en sort toute nue, avec de longs cheveux d'or. Une ruse ingénieuse lui a donné l'idée de vaincre ainsi "l'embûche de son destin ». L'étrange fiancée est acceptée par le peuple car elle ressemble aux représentations religieuses d'Ève, d'Agnès et des vierges martyrs, l'encens lui sert de voile pendant la cérémonie. La douce bergère a ainsi brisé la malédiction, il lui arrive de mettre chacune des premières robes des épouses défuntes ou sa robe de paysanne, mais elle n'a aucun vêtement propre qui puisse fixer le désir et conduire au meurtre. Le promeneur se demande finalement si la paysanne de la chaumière qui a fait surgir son récit ne serait pas cette dernière épouse.

Les images initiales qui s'accumulent créent chez Régnier une atmosphère propice à l'émergence d'un conte, suscitant une seconde scène énonciative où la légende naît d'abord des suggestions de la promenade et du paysage et de quelques mots allusifs, bien plus, les descriptions des épouses et des robes se révèlent contenir le motif et la fable même du récit: elles sont de la même étoffe que la légende réinventée par Régnier. La relation particulière entretenue par Barbe bleue et sa série d'épouses n'a plus aucun lien avec le mystère de la chambre close et la transgression de l'héroïne, mais s'explique entièrement par l'effet des robes de chacune de ces femmes, qui focalisent l'attrait érotique et finissent par rendre la personne superflue. La contemplation esthétique remplace définitivement le rapport au corps. La disparition de chaque épouse permet de lui substituer une autre avec sa propre robe : l'époux ne tue pas par goût du châtiment ou inclination à la violence, mais pour le plaisir d'une collection de robes qui peuvent rester en même temps des substituts de la femme ${ }^{20}$. Le déploiement des descriptions exhibées par Régnier, leur disposition en listes serrées, la précision des notations sensibles, le recours constant aux synesthésies, la trame des détails pittoresques, la densité des figures de style, l'association des beautés de la nature et de l'art, font entrer le lecteur dans l'expérience du héros, le rendent en quelque sorte complice de son goût criminel: son expérience de contemplation coïncide exactement avec celle de l'écrivain et du lecteur esthètes. Ils se laissent absorber par les représentations de la réalité sensible, au point de négliger la vie et d'éliminer leur partenaire. Du moins est-ce un univers d'hommes qui liquident les 
femmes en captant ce qui leur est propre, un mundus muliebris. La bergère qui clôt la série de façon pacifique est une femme de la nature, elle échappe à l'attrait du luxe et des biens et elle sauve le mari d'une espèce d'obsession, qui est aussi celle de l'écrivain et d'une écriture symboliste fascinée par le pouvoir expressif de l'image, par ses suggestions indéterminées, comme condensées elles-mêmes dans la robe couleur de l'aube et du crépuscule ${ }^{21}$.

Le récit de «La Belle au bois rêvant » de Catulle Mendès ${ }^{22}$ aboutit à une conclusion très voisine : la belle réveillée par le prince dit préférer retrouver les songes de la nuit et refuse de suivre le prince. Sa brève réponse évoque tout ce que lui apportent ses rêves et qui n'existe que par l'entremise de l'écrivain et de son style: la littérature vaut mieux que la vie!

Un écrivain contemporain, Amélie Nothomb, a modernisé «La Barbe bleue ${ }^{23}$ », en en faisant un court roman parfaitement vraisemblable, elle a conservé l'intrigue de Perrault, avec en son cœur le cabinet secret et la mort finale du héros. Mais dans sa transposition, elle a donné une place centrale à l'innovation de Régnier. Notons qu'une modernisation précédente de "Barbe bleue", due également à une romancière de grand talent, Angela Carter, "The Bloody Chamber» (qui donne son titre au recueil constitué seulement de reprises de contes classiques, de Perrault, Leprince de Beaumont et Grimm, avec l'interférence d'une légende du loup garou plutôt liée au cinéma contemporai ${ }^{24}$ ), avait placé l'action à la fin du $\mathrm{XIX}^{\mathrm{e}}$ siècle dans un esprit imprégné de l'univers symboliste. Le rapport au réel est entièrement esthétisé et fait appel à la mémoire savante du lecteur, évoquant les vampires, Ève et Caïn, l'œil de Dieu, la boîte de Pandore, les Mille et Une Nuits, le satyre, la méduse, «La Belle et la Bête ». Des références plus spécifiques à l'art incluent Huysmans, Wagner et Tristan, Debussy, Redon, Sainte Cécile, les primitifs flamands, Bruges la morte de Rodenbach, une série de tableaux (Ensor, Gauguin), une gravure érotique de Rops. Les robes de l'héroïne viennent des grands couturiers Worth et Poiret et viennent remplacer des vetements misérables: "Je portais donc, à l'Opéra, un sinueux drapé de mousseline blanche retenu par une cordelette de soie sous la poitrine» (p. 13); l'accent étant mis sur le "tour du cou de rubis". Les vêtements participent d'une séduction réciproque, l'homme qui les donne comptant sur eux pour éveiller la sensualité de la femme. Carter recourt à un bazar fin-de-siècle, encore présent dans son roman ultérieur Nights at the Circus (1984), pour transposer le lien établi par Perrault entre les objets luxueux offerts par la Barbe bleue et la transaction conjugale. La médiation symboliste - sur le modèle de Régnier - lui permet d'en dépeindre l'enjeu érotique, qui avait été introduit sous son aspect le plus brutal et retiré in extremis dans " Peau d'âne ». Carter se sert ensuite de la fable de "La Belle et la Bête", dans deux de ses contes, et de la fiction merveilleuse qui fait du monstre un prince charmant pour donner un tour fantastique à la quasi-équivalence de la robe somptueuse et du contact de la peau amoureuse. Dans «M. Lyon fait sa cour », l'héroïne, une fois rentrée chez elle, s'abandonne comme celle du «Cabinet sanglant » à l'agrément de la vie mondaine et, au retour d'une soirée, s'arrête devant un miroir. Elle contemple son visage, "that pearly skin of hers was plumping out» ("sa peau de perle prenait des allures vaguement replètes $\left.{ }^{25} »\right)$, et la description (on ne sait si cela correspond à sa propre vision) finit par voir dans sa peau la fourrure du chat: "Son visage était en train d'acquérir, au lieu de la beauté, un vernis de cette invincible joliesse qui caractérise certains chats de luxe exquis et pomponnés. » L'anglais lacquer s'applique à une surface brillante et dure qui serait dans un paradoxe conjointement celle de la femme et celle de l'animal, le mot vernis se 
prêtant à des acceptions plus métaphoriques : « Her face was acquiring, instead of beauty, a lacquer of the invicible prettiness that caracterizes certain pampered, exquisite, expensive cats. » (le dernier mot créant un effet de surprise perdu en français)

17 Le clignotement de la peau et de la fourrure d'abord suggéré comme une image va être le noyau de la conclusion de "M. Lyon fait sa cour", puisque l'héroïne, calquant ses gestes tendres sur ceux de la Belle chez Leprince de Beaumont, sent la fourrure du héros se transformer en peau. Symétriquement «La jeune épouse du tigre » voit sa propre peau, sous la langue active du tigre amoureux, se faire fourrure. La peau devient ainsi une sorte d'habit au titre d'enveloppe amovible. Dans «Peau d'âne » comme dans «Le cabinet sanglant » où l'orgasme bruyant de la Barbe bleue préfigure le plaisir qu'il prend à torturer et tuer ses épouses, le lien entre la robe et la peau prend un tour violent et même mortel, dont Régnier avait gardé la teneur en en changeant les termes, puisque chez lui la robe tient lieu de la femme devenant superflue; Carter loge le drame qui conjoint robe et peau dans la peau même qui se dédouble ${ }^{26}$, rendant possible une fin heureuse (comme dans le conte du XVIII ${ }^{\mathrm{e}}$ siècle).

Dans « Barbe bleue » de Nothomb, jouent aussi un rôle central les robes, les tissus dont elles sont faites et surtout leur couleur, conformément à ce qu'avait imaginé Régnier. L'attention à la couleur, singulièrement celle de l'or, est d'abord introduite dans le repas que l'hôte prépare à sa colocataire (p. 24), puis elle s'impose dans un entremets et avec le champagne (p. 36-37 et 42), reliés à l'extase amoureuse. Le héros expose (p. 60) sa passion pour les tissus et leurs couleurs et avoue qu'il a fabriqué la "superbe jupe » $\mathrm{du}$ " plus beau velours doré » offerte à l'héroïne. Il partage ensuite les penchants du Barbe bleue de Régnier et explique qu'il faut à chaque femme "un vêtement particulier » et présente la liste de ceux qu'il a conçus pour chacune des femmes dont les noms défilent aussi (p. 62-63). La narratrice établit le lien avec «Peau d'âne » et la robe couleur du jour. Dans leur conversation, le Barbe bleu moderne se réfère aux " couleurs" portées par la princesse de Clèves et aux rubans jaunes qu'elle enroule rêveusement autour de sa canne! (p. 82). L'héroïne découvre que le cabinet privé (et non fermé à clef: c'est la variation de cette version de l'histoire) est une chambre froide où sont affichées les photos des épouses qui y sont mortes de froid, revêtues chacune de leur robe particulière. Le héros livre la clef de son secret : « La couleur n'est pas le symbole du plaisir, c'est le plaisir lui-même. » Le vêtement capte dans sa texture singulière la couleur et en submerge la femme. La mort métaphorique de l'être sur la photo est, selon la logique du conte de fées, devenue un sens propre, et la photo conserve la couleur et le toucher de la robe. Le cabinet ne contient pas des cadavres mais une exaltation érotique de la femme qui implique son remplacement de chair par une enveloppe de couleur, la superficie de la photo venant donner sa durée et sa « laque » (selon le terme de Carter) à la superficie du vêtement.

On voit ainsi, au terme de ce défilé de robes, dans ces trois moments principaux, des changements dans l'appréhension de la robe, dans les valeurs qu'elle prend, dans son rôle dramatique, dans l'attention à sa matérialité sensible, qui correspondent à des différences dans le tracé du récit et dans les perspectives narratives, les modernes depuis Andersen choisissant de nourrir le conte des innovations venues de la fiction romanesque. Changent aussi le lieu des émotions dont la robe est le moteur, et surtout leur distribution, par conséquent les différents registres dont elles relèvent. Revenons à Perrault. D'emblée il met en avant la prouesse technique qui donne à la splendeur produite la marque d'une origine royale. Perrault fait ainsi apparaître très tôt le 
rapport de pouvoir et de violence inhérent aux quatre robes de l'héroïne (puisque le roi compte grâce à elles soumettre sa fille à l'inceste, et qu'elles aident la fille à y échapper puis lui font subir une autre persécution, venue du bas et non plus du haut). Cette multiplicité devient un instrument de libération, dans la mesure où l'alternance de la robe et de la peau d'âne empêche que l'une ou l'autre définisse l'identité de la jeune fille. Un moment, la robe, toute imprégnée de corruption tragique, devient un instrument de séduction décidément érotique : elle ne serait là que pour être enlevée et remplacée par la peau, la vision faisant alors place au toucher. Resurgirait ainsi la violence initiale par dissymétrie maximale entre celle qui porte et celui qui enlève la robe grâce à un pouvoir (de mâle et de prince) analogue à celui du père antérieurement. Une troisième voie se fait alors jour, conduisant à la fin heureuse : la robe sert bien de transition mais vers une identité qui n'est plus celle du sexe ou de la position sociale (souillon et princesse), mais celle de l'âme c'est-à-dire celle du Créateur. Marivaux modifiera cette reconnaissance : l'identité spirituelle procède d'une rencontre de deux subjectivités, elle dépend étroitement de la façon dont l'autre me regarde. Marivaux développe explicitement ce que Perrault avait suggéré : ce qui est infiniment d'un autre ordre que la robe, l'âme, ne saurait être perçu sans elle. La robe exalte donc contradictoirement un corps désirable et une âme admirable.

Régnier s'arrête à cette robe et à son agrément. Le héros ne renonce pas au plaisir érotique par respect pour une essence supérieure (l'âme), mais parce que la robe lui procure une jouissance plus intense : il fait l'économie de son propre corps parce qu'il est entièrement soumis à une attirance pour la beauté de la robe portée par la femme. La femme devient un auxiliaire de la robe et non l'inverse: tel est le renversement opéré par Régnier. La bergère dans les contes incarne la nature : ici, s'offrir nue, sans robe, empêche la substitution esthétique, la bloque plus qu'elle ne la remplace car le conte ne dit pas grand-chose des sentiments du mari à l'égard de la jeune épouse.

Nothomb reprend le récit symboliste et en transpose très fidèlement la logique qui a remplacé la femme par la robe et sa couleur. L'individualité de la femme est ramenée à ce que la société et le luxe moderne en font : une certaine manière singulière de choisir et de porter un vêtement. Régnier associait cette sorte de déshumanisation par l'esthétique au bric-à-brac pittoresque d'un romantisme exténué, Nothomb astucieusement le remplace par la photographie telle qu'interprétée par Roland Barthes dans La Chambre claire. Carter transforme encore le rapport de la femme à la robe. Perrault voulait que la robe serve seulement d'intermédiaire à une union spirituelle, le prince et le père à une union charnelle (chez Marivaux, $\mathrm{M}^{\text {me }}$ de Miran la mère entre dans le premier parti, le fils Valville dans le second, encore qu'il respecte une jeune fille qu'il aurait pu surprendre violemment nous dit la narratrice, il agit donc plutôt comme le prince de Perrault). Carter attribue à sa Barbe bleue une motivation sexuelle simple, mais c'est dans les adaptations voisines de « La Belle et la Bête » qu'elle donne à la robe une pleine dimension érotique en dédoublant la peau en fourrure, et donc en faisant de la peau une enveloppe qu'on peut enlever et remplacer (sans doute celle-ci se modifie-t-elle dans l'amour et devient différemment sensible). Carter est la plus fidèle à la richesse du propos de Perrault (à travers Leprince de Beaumont et « La Belle et la Bête »), dans la mesure où elle conserve l'articulation entre le mouvement du désir amoureux et les situations de pouvoir, d'oppression sociale et de menace de l'intégrité de l'autre (auquel s'oppose ce que la jeune épouse du tigre appelle «le respect »). Perrault montrait la tension entre l'espace chrétien, celui du pouvoir et celui de l'appétit, en les éclairant par les rapports à la fois d'union et de divergence entre le 
corps et l'âme, ce monde et l'autre monde; Marivaux pourtant pieux ramène la métaphysique à l'humanité fondée en soi par la reconnaissance de l'autre; Régnier introduit dans une relation à la fois érotique et sociale une distance esthétique qui est aussi celle de la littérature comme fière et malheureuse de sa capacité à représenter le monde ; Carter et Nothomb réintroduisent le corps présent mais écarté par Perrault et par Régnier (Marivaux comme toujours conserve les deux propositions contradictoires d'une présence et d'un effacement du corps) avec deux échappatoires complètement différentes. Régnier commence par la religion et conclut par un mariage à l'église mais c'est un décor à l'ancienne qui rappelle ce qu'il néglige, comme Marivaux avait placé la scène de première séduction de La Vie de Marianne dans une église : il est difficile d'être plus offensif dans la transgression. L'introduction du corps reste chez Nothomb liée à l'esthétique qui finalement remplace l'étreinte, mais dont le désir subsiste comme principe de répétition de l'union, condamnée à être finalement décevante et remplacée par l'art : Nothomb reprend à peu près l'explication de la série de Régnier, en dehors de tout goût pervers pour le meurtre. Ce changement (dans le prolongement du symbolisme, comme chez Carter) est associé aux mœurs modernes plus encore présentes encore dans la version de "Riquet à la houppe " que la romancière a publiée juste après, où la laideur du héros en fait un tombeur vaguement ennuyé des demandes répétées des femmes à son corps difforme. Carter fait résonner dans le moment fin-desiècle le discours freudien, cela l'amène à une invention très originale où la peau est aussi un vêtement, double et mobile. On pourrait considérer que la version de Marivaux s'approprie de façon très singulière la logique sensualiste, ou plus exactement adapte à cette logique le message de Perrault. Celui-ci et ses amies conteuses ont fondé le genre du conte de fées sur la confrontation du présent le plus contemporain au passé le plus lointain et le plus archaïque, qui peut toujours resurgir dans des croisements imprévisibles. La multiplicité des robes de Peau d'âne et leur présence au long de l'histoire, en particulier dans ses espaces successifs, en font le support matériel de cette compénétration des temporalités et des univers correspondants. Les deux parures contradictoires de l'héroïne sont portées par la sauvagerie primitive qui se retrouve dans le monde ancien de l'âne aurifère comme dans la ferme du jour, chez les valets comme chez le prince tenté par la violence, mais au même moment la robe introduit une dimension métaphysique qui échappe au temps avant que le temps ne soit rétabli dans le mariage final. Les avatars symbolistes de «Barbe bleue » intensifient le rapport sensuel aux robes tout en préservant le jeu sur les temporalités, sur l'éloignement et la proximité qui valent aussi bien pour le temps présent que pour le temps passé. L'élément concret - ici les robes des hérö̈nes - ne peut donc être ramené à son unité objective, puisqu'il se multiplie par les valeurs elles-mêmes diverses dont il est investi et, si l'on veut, les perspectives que le récit ménage et intègre, et donc les investissements affectifs, admiration, violence, désir, etc. dont il est susceptible. L'expérience sensible se révèle ainsi non dans sa simplicité phénoménologique, comme le roman du xix ${ }^{e}$ siècle la traite souvent, mais comme un objet littérairement construit, et dont le cubisme nous donnerait une image plus récente. 


\section{NOTES}

1. Dans le sens exploré par A. Gaillard dans Fables, mythes, contes: l'esthétique de la fable et du fabuleux (1660-1724), Paris, H.Champion, 1996 et par J.-P. Sermain dans Métafictions, Paris, H. Champion, 2002.

2. Voir J.-P. Sermain, «Regards furtifs sur la fiction (1646-1734), Chapelain, Huet, Galland, Marivaux, Prévost ", Études françaises, vol. 49, n 1, 2013, p. 13-22.

3. Dans Opuscules critiques, éd. A. C. Hunter, Droz, Paris, p. 63. Curieusement cet éditeur a peu d'estime pour l'auteur et ses idées. Cité par R. Démoris, Le Roman à la première personne, Paris, Armand Colin, 1975, p. 19.

4. Je suis ici l'enseignement de Roger Zuber dispensé en 1978 à Nanterre et dont il a nourri sa magnifique édition des Contes de Perrault pour l'Imprimerie nationale (1987, dont la préface a été reprise dans les Émerveillements de la raison, Paris, Klincksieck, 1997).

5. Voir l'étude très complète de M. Blanco, Les rhétoriques de la pointe. Baltasar Gracián et le conceptisme en Europe, Paris-Genève, H. Champion, coll. «Bibliothèque de l'Humanisme et de la Renaissance », 1992.

6. Joseph Addison en 1711 prend pour exemple le conte de fées pour définir la poétique de l'horreur agréable, suivant sans le savoir sans doute Marino, voir J.-P. Sermain, «A pleasant kind of horror ", dans J. Berchtold, R. Démoris et C. Martin (dir.), Violences du rococo, Pessac, PUB, 2012, p. 29-40.

7. H. Merlin, « Un siècle classico-baroque ? ", XVII ${ }^{e}$ siècle, 2004/2, p. 163-172.

8. L'expression vient de Perrault, dans la préface des contes en vers, pour qualifier les « fables » populaires dont ils sont nourris.

9. Le texte laisse cet émoi dans l'ombre, on ne sait si le vieux dévot est séduit par Marianne ou profite seulement d'une occasion offerte par la faiblesse d'une fille des rues sans famille: jolie proie après tant d'autres? Marivaux suggère le système mais ne veut pas trop assombrir son roman ni tomber trop dans les bas-fonds.

10. Toutes les références sont empruntées à l'édition de Jean-Pierre Collinet des Contes pour la collection Folio, en 1981. «Peau d'Âne » occupe les pages 95-116. On s'y reportera.

11. De façon remarquable, les frères Grimm se saisissent des éléments concrets du "Petit poucet " pour en tirer des effets sensibles à la fois visuels et émotifs. Ainsi, le héros, qui a entendu ses parents préparer son assassinat et celui de sa sœur, se retourne régulièrement lorsqu'ils sont traînés vers la forêt par le père, et se justifie ainsi : «Je regarde mon petit chat blanc qui est posé au haut du toit et qui veut me dire adieu », à quoi le père réplique : «Ce n'est point ton petit chat, c'est le soleil du matin qui brille sur la cheminée. » La scène se répète lors de la seconde tentative d'assassinat : «Je regarde mon petit pigeon qui est posé sur le toit et qui veut me dire adieu », à quoi le père répond : «Ce n'est pas ton petit pigeon, c'est le soleil du matin qui brille sur la cheminée. » Abandonnés une première fois dans la forêt, les enfants attendent «que la lune soit levée ", et Hänsel «suivit les petits cailloux blancs qui brillaient comme des pièces d'argent toutes neuves » jusqu' « au point du jour ». Abandonnés une seconde fois dans la forêt, ils ne voient rien briller dans la nuit, et le lendemain « [q]uand vint midi, ils virent un joli oiseau, blanc comme neige, perché sur une branche » et ils « le suivirent ». Les Grimm introduisent des images et des lumières et les intègrent au récit forgé par Perrault (Grimm, « Hänsel et Gretel », dans Les Contes de Perrault dans tous leurs états, éd. A. Colognat et M.-C. Delmas, Paris, Omnibus, 2007, p. 939-948). Grimm en revanche abandonne la «petite lueur, comme d'une chandelle » qui perce la nuit de l'horreur et annonce au Petit poucet et à ses frères un refuge, celui de l'ogre ! 
12. Marina Warner fait de l'expérience des limites le propre du conte de fées, dans From the Beast to the Blonde. On Fairy Tales and their Tellers, New York, Noonday Press, 1999.

13. Dans la pièce qui porte ce nom, acte I scène 4 .

14. Voir le bel article de R. Démoris, "Du littéraire au littéral dans "Peau d'Âne" ", Revue des Sciences Humaines, t. XLIII, $\mathrm{n}^{\circ}$ 116, avril-juin 1977, p. 261-279.

15. L'intérêt de Perrault pour ce signe sensible qu'est la robe nourrit d'autres contes comme «Cendrillon » et « La Belle au bois dormant».

16. Voir J.-P. Sermain, «De la robe de Peau d'Âne à la parure de Marianne: Marivaux et l'expression de soi », dans B. Guion, M.-S. Seguin, S. Menant et P. Sellier (dir.), Poétique de la pensée, en hommage à Jean Dagen, Paris, H. Champion, 2006, p. 875-886. Sur le viol dans le conte de fées, voir J.-P. Sermain, « L'aubaine et la réparation. Deux figures du viol dans les nouvelles et les contes de Cervantès à Diderot ", Tangences, $\mathrm{n}^{\circ}$ 114, 2017, p. 45-60.

17. Voir sur ces relations et les choix symbolistes les deux livres de J. de Palacio, Les Perversions du merveilleux (Paris, Séguier, 1993) et Les Métamorphoses de Psyché (Paris, Séguier, 1999) ; ainsi que E. Stead, Le Monstre, le singe et le fœetus, tératogonie et décadence dans l'Europe fin-de-siècle, Genève, Droz, 2004.

18. La nouvelle a été consultée et citée dans la très belle édition de B. Vibert avec la collaboration de M. Beghin, Contes symbolistes, Grenoble, ELLUG, 2011, vol. 2, p. 432-446 (voir aussi les riches introductions) ; autre édition dans Les Contes de Perrault dans tous leurs états, éd. citée, p. 537-549.

19. P. 439, on peut penser aux robes de Fortuny, aimées entre autres par Proust.

20. L'ordre social est menacé par la succession des épouses, mais il incite au contraire à multiplier les robes, et à transformer le château en une vaste garde-robe. Barbe bleue est un héros moderne prenant à la lettre la fétichisation de la marchandise.

21. C'est ce qu'annonce Henri de Régnier dans l'avis «Au lecteur» de La Canne de Jaspe (Contes symbolistes, éd. citée, p. 221) à propos de son livre : « S'il présente un sens inattendu au-delà de ce qu'il semble signifier, il faut jouir de ce surcroît à demi intentionnel sans y exiger trop de suite et en le considérant comme né fortuitement des concordances mystérieuses qu'il y a, malgré tout, entre toutes choses »: le sens procède en partie d'une intention imprécise et du hasard des correspondances réelles. Voir M. H. Abrams, The mirror and the lamp: romantic theory and the critical tradition [1953], New York, The Norton Library, 1958 ; le livre important de F. Kermode sur la notion d'expression, Romantic image [2001], Londres, Routledge Classics, 2012; le livre de P. Labarthe, Baudelaire et la tradition de l'allégorie, Genève, Droz, 1999; et sur cette même notion d'expression, mais dans une perspective très différente, celui de J. Rancière, La Parole muette, essai sur les contradictions de la littérature [1982], Paris, Hachette, coll. « Pluriel », 1998.

22. Paru dans Les Contes du rouet en 1885 , lu dans Les Contes de Perrault dans tous leurs états, éd. citée, p. 264-268.

23. A. Nothomb, Barbe bleue, Paris, Albin Michel, 2012, rééd. Le Livre de Poche, 2015.

24. A. Carter, The Bloody Chamber [1979], Londres-New York, Penguin Books, 1981, rééd. 1993 ; La Compagnie des loups, trad. J. Huet, [1985], Paris, Points, 1997.

25. Éd. citée, respectivement p. 48 et p. 100.

26. Voir à ce sujet le livre de D. Anzieu, Le Moi-peau, Paris, Dunod, 1985. 


\section{RÉSUMÉS}

Pour voir comment le conte classique traite «l'univers sensible des contes », cet article isole une composante matérielle essentielle du récit de "Peau d'Âne», les trois robes de l'héroïne, et envisage d'abord la manière dont Perrault le traite. Est ensuite vu comment cet élément devenu topique vient se greffer sur une adaptation par Henri de Régnier de « Barbe bleue », puis sur deux adaptations contemporaines de «La Belle et la Bête " par Angela Carter et une dernière version de «Barbe bleue » par Amélie Nothomb. Ces divers modes d'évocation du vêtement merveilleux impliquent différents engagements des personnages et du lecteur. Ils entrent dans les conceptions des rapports amoureux et de l'identité forgées par ces divers contes classique, symboliste et contemporains. Il semble ainsi que ce soient des modèles anthropologiques qui déterminent les changements dans le sentiment du réel davantage que des révolutions épistémologiques.

To answer the question of the treatment of the material world in fairy tales, this paper first considers how Perrault represents the three marvelous dresses of "Peau d'Âne" and which part they play in her relationship with her father and her lover. Then, this paper examines how the French symbolist poet Henri de Régnier deals with the topic of the dresses in his adaptation of "Barbe bleue"; and finally how two women authors, Angela Carter and Amelie Nothomb, have recently adapted the works of Perrault and Régnier and proposed their versions of the same theme in their own versions of "La Belle et la Bête" and "Barbe bleue". Therefore, it is so suggested that the changes in the ways of rendering these dresses are not due to epistemological revolutions but to variations in anthropological conceptions.

\section{INDEX}

Mots-clés : Charles Perrault, contes, adaptation, Henri de Régnier, Amélie Nothomb, Angela Carter

Keywords : Charles Perrault, tales, rewrite, Henri de Régnier, Amélie Nothomb, Angela Carter

\section{AUTEUR}

JEAN-PAUL SERMAIN

Université Sorbonne nouvelle - Paris 3 